\title{
The Research and Development of the Amphibious Disaster-Resistant Submersible Pump with Dual Pump and Dual Motor
}

\author{
Feng Yicheng* \\ Department of Electrical Engineering, Tsinghua University, Peking, 10084, China
}

\begin{abstract}
This thesis introduces a kind of horizontal wet electric submersible pump unit with both submersible and nonsubmersible operational models which can be installed permanently. Then, it analyses the structural features, operating principles, main component performance and rotor system. A series of technical advantages show that this kind of submersible pump has a bright application prospect in conventional drainage and disaster-resistant emergency drainage of deep mines.
\end{abstract}

Keywords: Amphibious, disaster-resistant, dual pump and dual motor, submersible pump.

\section{INTRODUCTION}

According to the two hundred and seventy-third regulation of the new edition of Coal Mine Safety Regulations, when the hydro geological conditions are complicated or the mine is at a risk of having water flooded wells, the water proof gate should be set around the shaft station or the other independent power supply system should be installed for implementing the basis of normal drainage around the bottom, where the drainage capacity is not less than the maximum yield of submersible pump [1]. This regulation indicates that the submersible electric pump should be fixed from the bottom so that it can be stable for a long time. The traditional submersible pumps must sink when running and it used in a pump off the plane on the vertical installations [2]. These traditional submersible pumps used to resist disaster at the bottom for years require setting of the water at the bottom in a way which increases the quantity of mines and the difficulty of installment. Moreover, they are more likely to be corroded severely and buried by sludge due to being placed in the mine water for a long term. Based on this, this dissertation introduces a horizontal installation of Amphibious Disaster-resistant submersible pump which can not only drain off water in mine like a normal pump, but can also be used as a submersible pump to support disaster and disaster relief drainage in the mine. The amphibious pump usually does not have to sink into the water for avoiding the mine water erosion of the pump body which is also convenient for daily maintenance.

\section{OVERALL STRUCTURE AND WORKING PRINCIPLE}

\section{Structural Features}

The amphibious disaster-resistant submersible pump's structure is shown in Fig. (1). The pump is composed of the two same specifications of horizontal pumps and electric

\footnotetext{
*Address correspondence to this author at the Department of Electrical Engineering, Tsinghua University, Peking, 10084, China;

Tel: 13099208668; E-mail: tsinghuafyc@icloud.com
}

motors. Horizontal pump is fixed on the pump shaft through the pump shaft itself and as well as through water outlet, water inlet, mesomere, blast hole, guide vane and impeller. In addition, the pump shaft passes through the position of pump shell's tail in order to be sealed mechanically. The right and left positions of horizontal pump are symmetric and concentric whilst the pump shaft end at the middle of horizontal pump is connected permanently with the help of the coupling shaft. The electric motor is comprised of the motor shaft, motor case, front cover, mechanical sealing device, stator assembly, rotor assembly, thrust bearing, adjusting bag, back cover and other parts [3]. The motor shaft and rotor assembly have access to the internal motor and are as well supported by the front bearing and the rear bearing. There is a mechanical seal device located between the front case and the front bearing and the adjusting bag is fixed on the back cover. Therefore, the shafts of these two horizontal pumps are respectively connected with a motor by a coupling. The motor casing is fixed on the mounting by a screw, and there is an outer channel setting on the motor casing. In this way, the inlet can be connected with the outer channel on the motor casing by an adjusting bag whilst the outer channel is connected with the suction of horizontal pump by a clamp, and the horizontal pump outlet is connected with whole drainage pipe by three links. The submersible motor is installed on the friction material carrying friction resistance on both ends, which is used to eliminate the residual axial force of manufacturing errors.

\section{Operating Principle}

When the amphibious disaster-resistant submersible pump is running, the two pumps arranged symmetrically can be driven by two sets of the same type of submersible motor so as to balance the pump axial force produced by the runtime [4]. The mine water from water inlet flows through the web of dome, casing pipe, spherical shell in sequence, and then enters into the horizontal pump. In the meantime, the water flows within the web of the cooling water channel into the web of pipeline internal flow in order to sufficiently exchange the heat with the motor, for guaranteeing the normal operation of motor driving the pump. Besides, when the mine water inflow increases dramatically and even 


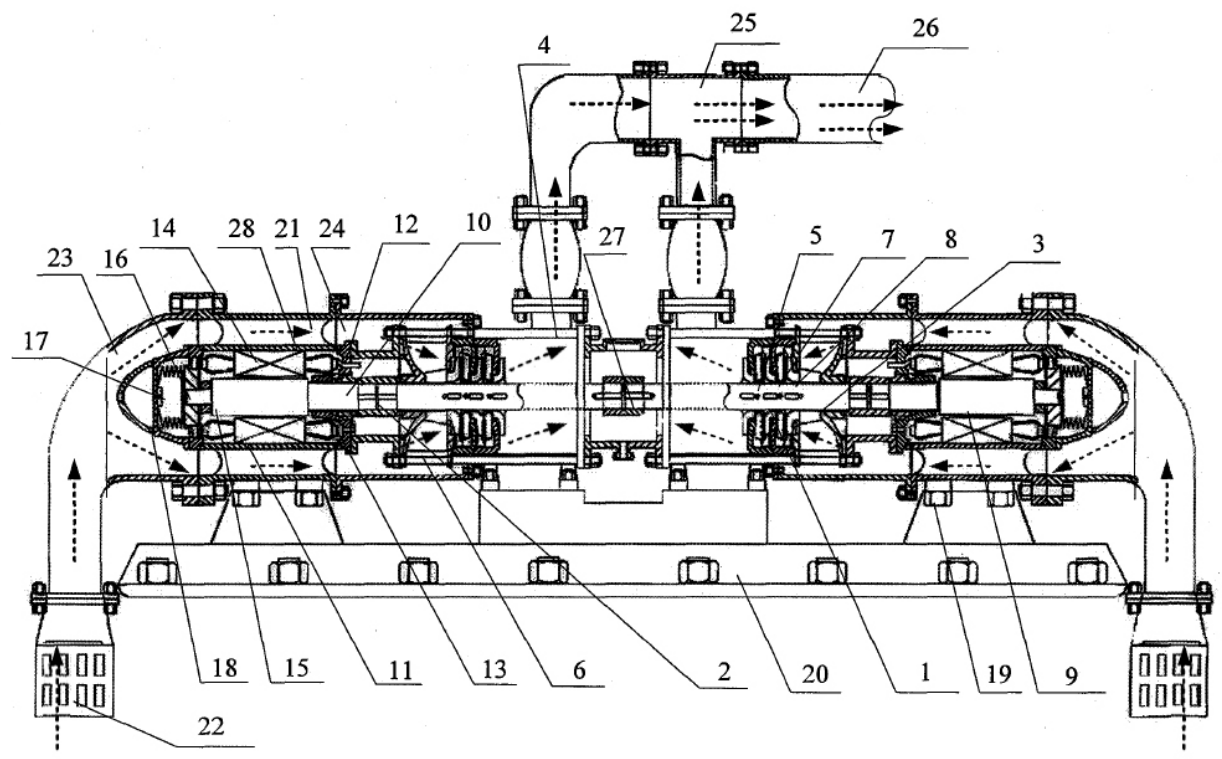

1 - Horizontal Pump; 2 - Coupling; 3 - Pump bearing; 4 - Water outlet; 5 - Mesomere; 6 - Suction; 7 - Guide vane; 8 - Impeller; 9 - Electric motor; 10 - Motor shaft; 11 - Motor case; 12 - Front housing; 13 - Mechanical seal device; 14 - Stator module; 15 - Rotor assembly; 16 - Thrust bearing; 17 - Adjust bag; 18 - Back cover; 19 - Screw; 20 - Fixed bearing; 21 - Web of pipes; 22 - Water inlet; 23 - Fairwater; 24 - Clamp; 25 - Three Links; 26 - Total drainage pipeline; 27 - Coupling; 28 - Web of cooling channel.

Fig. (1). Amphibious type submersible electric pump overall structure diagram.

exceeds the mine drainage ability, the water level rises sharply. If the amphibious pump can be started before being inundated, it can serve as a normal drainage pump and can assist in the mine drainage system's normal work, effectively increasing the mine drainage ability and inhibiting or delaying the time of mine flooding so that the staff underground can have more time to escape the mine well and the rescue follow-up can be conducted. On the contrary, if the water inflow has increased continually leading to the mine flood and the entire amphibious pump is submerged in the mine water, the amphibious pump acting as relief submersible pump lays a foundation for subsequent drainage after accident in turn reducing the loss caused by the accident [5]. The flow direction of amphibious disasterresistant submersible pump is shown in the arrow direction of Fig. (1).

\section{STABILITY ANALYSIS AND PERFORMANCE REQUIREMENTS}

\section{The Rotor System Characteristics Analysis}

By comparing with ordinary vertical submersible pump, the rotor system is the key for evaluating amphibious pump horizontal running [4]. Therefore, it is necessary to analyze the characteristics of rotor load, critical speed and dynamic deflection.

The simplified model of amphibious disaster-resistant submersible pump with rotor system is shown in Fig. (2).

\section{Force Analysis of Rotor System}

The Amphibious disaster-resistant submersible pump belongs to horizontal multistage centrifugal pump. The load

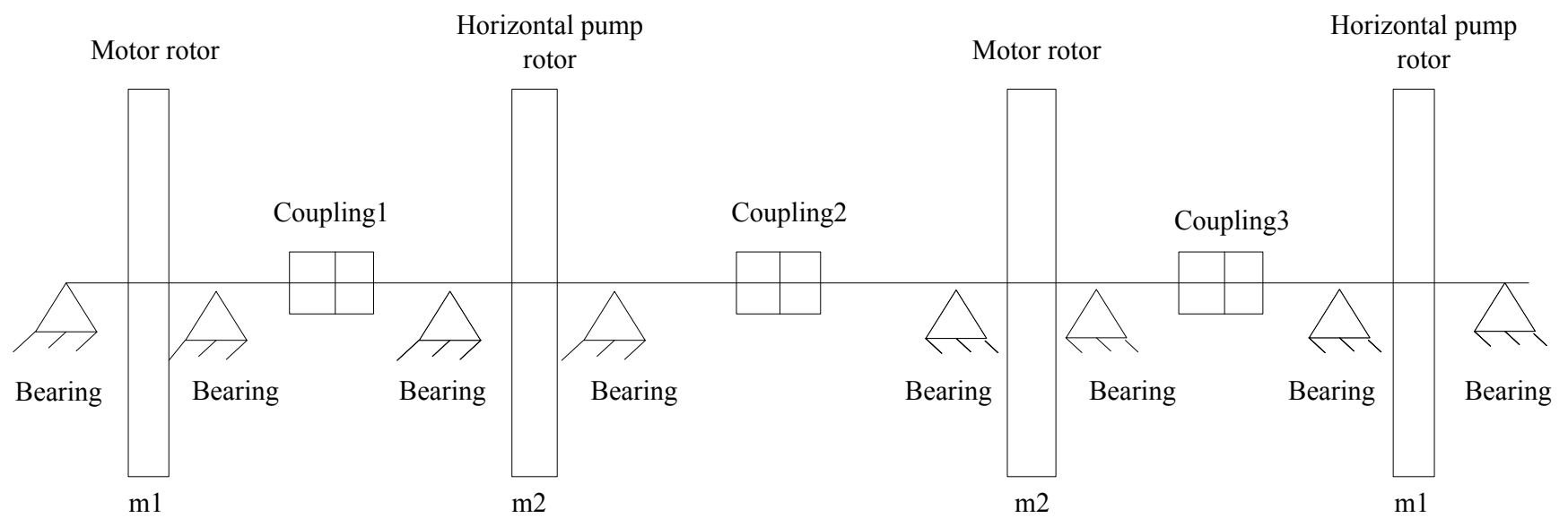

Fig. (2). Simplified model of amphibious disaster-resistant submersible pump with rotor system [5]. 
on the rotor system includes:

(1) The radial force, including the additional radial force on the impeller rotation due to the uneven distribution of pressure along the outer edge of the impeller, the centrifugal force resulting from the residual unbalance weight when the rotor system is rotating and the radial force produced by the weight of the overall rotor system.

(2) The Axial force mainly refers to the axial force produced by the imbalance of multiple impellers.

(3) Torque passing by the pump shaft. Radial force is balanced by the centripetal bearing, axial force is balanced by the thrust bearing, and the pump shaft balances the torque.

As the two structures of both right and left horizontal pumps of this kind of submersible electric pump are exactly the same, these can generate equal and opposite axial forces to offset each other so as to balance the axial force on the rotor system. At the same time, thrust bearing is installed at the end of the motor to balance the residual axial force. Therefore, when pumps are working, the axial force is especially important for the three kinds of loads added on the rotor system. It is also the key factor for influencing the stable operation of the pump.

\section{Critical Speed and Dynamic Deflection of Rotor System}

The impellers of two pumps in this device are fixed on the pump shaft and the influence of coupling and pump shaft's own weight can be ignored. The situation could be assumed that the impeller is in the middle level of pump shaft without being damped. The influence of the impeller's own weight on critical speed is investigated here.

The quality of the impeller $F_{c}=\frac{m u^{2}}{R}$ could lead the axis of horizontal axis to descend to point $\mathrm{C}$, as shown in Fig. (3). The static deflection $A_{0}$ is formed. Considering the gravity eccentricity of impeller $u_{c h \max } \leq \sqrt{\frac{g R(f-\tan \theta)}{f \tan \theta+1}}$, the distance of the impeller center $\mathrm{C}$ deviating from the bearings $\left(F_{c} \cos \theta+G \sin \theta\right) h_{g} \leq \frac{\left(G \cos \theta-F_{c} \sin \theta\right) B}{2} \quad$ (dynamic deflection of the axis) make the radius of the impeller gravity $\mathrm{W}$ centering rotation at point $\mathrm{C}$ to be $u_{c f \max } \leq \sqrt{\frac{g R\left(B-2 h_{g} \tan \theta\right)}{2 h_{g}+B \tan \theta}}+u_{c h \max } \leq u_{c f \max }$. The impeller center of gravity $\mathrm{W}$ rotates about the line connecting the bearing $\mathrm{O}$, the gravity center producing the centrifugal force is:

$$
\sqrt{\frac{g R(f \cos \theta-\sin \theta)}{f \sin \theta+\cos \theta}} \leq \sqrt{\frac{g R\left(B \cos \theta-2 h_{g} \sin \theta\right)}{2 h_{g} \cos \theta+B \sin \theta}}
$$

The change of the pump shaft is within the elastic range. Its force has direct ratio in relation with the strain, which is shown as: $f \leq \frac{B}{2 h_{g}} \cdot \frac{1+\operatorname{tg}^{2} \theta}{1-\operatorname{tg}^{2} \theta}$

$\sup (X)_{i}<s \times d_{i}$ - Rigidity coefficient of the pump shaft

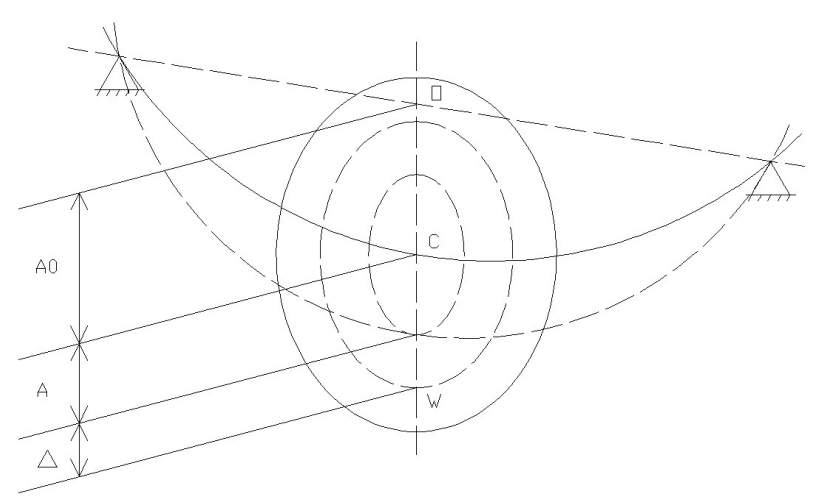

Fig. (3). Simplified model of horizontal pump with rotor system.

Comparing the formula (1) with (2), the result is:

$A=\frac{m\left(A_{0}+\Delta\right) w^{2}}{K-m w^{2}}$

When the angular velocity of pump shaft is increased to the angular frequency, it means that the dynamic deflection $A=\infty$. The critical angle frequency $(A=>B)$ :

$w_{c}=\sqrt{\frac{K}{m}}$

The critical speed $\operatorname{Sup}_{(A \cup B)}>$ min_sup is:

$n_{c}=\frac{30 w_{c}}{\pi}=\frac{30}{\pi} \sqrt{\frac{K}{m}}$

Based on the formula above, even if there is no gravity eccentricity shaft in rotor pump, which means that $\Delta=0$, the angular frequency vibration of the rotor could still be ensured as $w_{c}$ on the base of the quality of the rotor $C V$ and the rigid coefficient $A=>B$. So, from the perspective of the angular frequency vibration of the rotor, the rotor critical speed is not related to the eccentricity.

Merging the formulas of (3), (4), (5):

$A=\left(\frac{w^{2}}{w_{c}-w^{2}}\right)\left(A_{0}+\Delta\right)=\left(\frac{n^{2}}{n_{c}-n^{2}}\right)\left(A_{0}+\Delta\right)$

according to (6), if $n<n_{c}$, the dynamic deflection $A$ increases with the increase in rotational speed; If $n<n_{c}$, the dynamic deflection $A$ decreases with the increase in rotational speed (absolute value); If $n=n_{c}$, the dynamic deflection $A$ is maximum.

The submersible electric pump manufacturing error and setting error of rotor system and other factors lead to the forced vibration of submersible electric pump rotor system due to the unbalanced eccentric quality. 
In the actual operation, in addition to the eccentric quality caused by pumps forced vibration, the other influenced factors of dynamic deflection $A$ are due to the misalignment issue of three couplings linking with the four axes, the gap issue of shaft and bearing's cooperation. It is shown as follows.

$$
A=\gamma_{1}\left(\frac{w^{2}}{w_{c}-w^{2}}\right)\left(A_{0}+\Delta\right)=\gamma_{1}\left(\frac{n^{2}}{n_{c}-n^{2}}\right)\left(A_{1}+\Delta\right)
$$

In this formula above, $\gamma_{1}$ is the influence coefficient of dynamic deflection due to the misalignment rotor and the fitting clearance.

Therefore, the rotor system must be checked carefully before assembly according to the static and dynamic balance so as to enable the eccentricity $\Delta i$ of the gravity center $w_{i}$ of each of the impeller close to zero and for making the dynamic deflection $A_{i}$ and centrifugal force smaller to slow down the bearing load of radial shaft. It is necessary to strictly control the problems regarding the misalignment rotor and the fitting clearance.

\section{Performance Requirements of the Main Components}

In order to estimate the horizontal running with high flow, high lift and high efficiency, the main components of the amphibious disaster-resistant submersible pump should meet the following requirements by considering the installation conditions, working conditions and other factors [6].

\section{Water Pump}

The two water pumps should be controlled by the same switch which ensures the start and stop of two motors at the same time and the relative rotation. Water enters into the pump body respectively from the water inlet of the two pumps. Then, the working of impeller increases the energy. After this, water emerges from the water outlet and flows through the coupling flange and check valve to lifting pipe. There are two bearings on the each side of the water pump shaft and the check valve is installed on the total drainage pipeline, which avoids the water pump reversal caused by the back-in water when parking.

\section{Pump Case}

Pump suffers great pressure when working. So, it should be manufactured by using the high strength ductile iron. Parts should be evaluated through a hydrostatic test according to the provisions of the state standard. Gasket and seal gum are placed between the level shells to prevent leakage to ensure the good performance of the pump. Due to the quality of water, both the internal and external of pump case should use antirust processing.

\section{Impeller and Guide Blade}

The Impeller is composed of the front cover, back cover, blades and impeller ring. The advanced casting method is adopted to ensure the geometrical shape of the impeller and highly furnished surface. The smooth operation can be guaranteed by conducting the static balance test after the processing completion.
Both the Impeller and guide blade are prepared with the special material or undergo processing of surface properties so as to ascertain the performances of wear-resisting and corrosion resistance. The stainless steel impeller ring is inlayed at the impeller seal.

\section{Pump Shaft}

The material of the pump shaft is $40 \mathrm{Cr}$ through thermal refining and qualitative processing. Generally, the chrome plated collar and interstage sleeve protect the pump shaft. The two pump shafts are connected with coupling.

\section{Coupling}

When the pump is launched, the overload phenomenon occurs during the process of dynamic load operation. The maximum torque in the shaft torque should be served as the calculation torque $T_{c a}$, with the torque formula [7] being:

$T_{c a}=K_{A} T$

In this formula, $\mathrm{T}$ is nominal torque $(N \cdot m) ; K_{A}$ is work situation coefficient. According to the characteristics of bigger pump shaft torque and moderate impact load, $K_{A}$ takes 1.9 .

\section{Bearing}

Horizontal dual pump has four radial bearings, lubricated by water. Lubrication bearing's water keeps on recycling by the pressure difference. All of them are made of tin bronze.

\section{Adjusting Ring}

This pump is the multiple-stage centrifugal pump, of which the assembly cumulative error and adjusting loop compensation error are great. The centerline of the impeller outlet is higher than the centerline of the vanes entrance (It is advisable to $0.5 \sim 1.0 \mathrm{~mm}$ ).

\section{THE MAIN INNOVATIVE POINTS}

Amphibious disaster-resistant submersible pump provides a new way of drainage in the mine disaster and disaster relief. By comparing with ordinary submersible pump, there occur some innovation points as follows:

(1) Using the same pump symmetrical layout, the structure can realize the axial force balance when running.

The fission horizontal installation of submersible motor used is convenient for transportation, installation, lifting and removal.

(3) It can be installed under mine near the main water storehouse for long year, which can not only be able to work as a normal mine drainage pump, but also can work as a submersible pump in the anti-disaster and disaster relief. When the mine water is gushing normally, it works as a dewatering pump to assist in the main drainage pump inside the pump house to drain away the water, improving the pump house's capacity of pump drainage. When the mine water inflow increases and even when accidents occur, it can contribute to the drainage for fighting disaster, saving valuable time for the miners out of the mine and reducing the probability of the accident of the flooded well. When the mine is submerged 
completely, this equipment can be converted to the submersible pump to contribute to the rescue and drainage, reconstructing the mine so as to reduce the damage caused by the accident [8].

(4) At ordinary times, it is not necessary to subduct into water that could monitor and observe the pump running status at any time to make maintenance convenient at any moment. Not only can the reliability of this pump be ensured, but also the difficulty and the cost of maintenance are reduced. It also enables the annual routine submersible pump commissioning not to be carried into water. At the same time, it does not need to be flooded in water as usual, which can avoid the silt, minerals and impurities erosion of submersible motor and submersible pump. In this way, the service life could be extended.

(5) The flexibility of amphibious pump is increased as the drainage pump, since the length of suction pipe can be extended.

(6) The same water pump can be shared with the underground drainage system in order to reduce the project amount of mine.

\section{CONCLUSION}

Amphibious disaster-resistant submersible pump not only provides a new draining way for the mine, but also confers the dual function for high-power submersible pumps, in terms of resisting and relieving disaster. Therefore, it provides the reference for the construction of Chinese coal mine disaster in deep draining system. Furthermore, it increases the scope of application of the power submersible pumps because of the development of amphibious disasterresistant submersible pump, offering the equipment and technique for constructing safe, efficient and modern mines.

\section{CONFLICT OF INTEREST}

The author confirms that this article content has no conflict of interest.

\section{ACKNOWLEDGEMENTS}

Declared none.

\section{REFERENCES}

[1] F. Lijie, G. Chuanchang, and Y. Wuzhou "The research of highpower submersible pump drainage operating mode", China Rural Water and Hydropower, vol. 8, pp. 94-95, 2003.

[2] F. Lijie, "A new pump combined operation mode", China Coal, vol. 28, pp. 46-47, 2002.

[3] H. Ming, and Y. Yongping, "Low-speed large-capacity submersible pump motor design practice", Electrical Machinery Technology, vol. 4, pp. 7-9, 2012.

[4] L. Yongqiang, and C. Hantao, "Advantages of diving pump for mine drainage in mine and problems of selection", Coal Mine Machinery, vol. 33, pp. 215-216, 2012.

[5] C. Weidong, G. Yi, W. Xiulan, and X. Rongjun, "Optimization design of submersible pump rotor system for coal mine based on Ansys", Journal of Drainage and Irrigation Machinery Engineering, vol. 30, pp. 159-160, 2012.

[6] F. Lijie, "The research of one thousand meters mine water disaster rescue key technology and equipment", Zhengzhou University, 2011.

[7] P. Lianggui, and G. Minggang, Mechanical Design. Higher Education Press, Beijing 2006.

[8] S. Qiaorui, Y. Shouqi, W. Chuan, and B. Hu, "Optimal design of submersible multistage pumps with low specific speed", Transactions of the Chinese Society of Agricultural Engineering, vol. 28, pp. 123-124, 2012. 